\title{
ACCIÓN CONJUNTA INTERGENERACIONAL (ACIG). DESCRIPCIÓN DE VARIABLES INTERVINIENTES
}

\author{
Alejandro Canedo-García \\ Universidad de León \\ acang@unileon.es \\ Jesús-Nicasio García-Sánchez \\ Universidad de León / Universidad de la Costa (Colombia) \\ jn.garcia@unileon.es \\ Deilis-Ivonne Pacheco-Sanz \\ Universidad de Valladolid \\ deilisivonne.pacheco@uva.es
}

Fecha de Recepción: 9 Marzo 2019

Fecha de Admisión: 30 Abril 2019

\section{RESUMEN}

Con el objetivo de conocer los diferentes tipos de actividades conjuntas realizadas por miembros de distintas generaciones se administró un cuestionario online a un total de2013personas. De estos, 608eran hombres y 1405 mujeres. El cuestionario fue diseñado tras una revisión de los instrumentos empleados en el campo de trabajo intergeneracional en diversos estudios nacionales e internacionales. De manera online se estableció contacto con los posibles participantes para informarles sobre el propósito del estudio y solicitar su participación. Los encuestados dieron su consentimiento informado a participar en el trabajo. Una vez extraídos y codificados los datos se realizaron los análisis descriptivos (tablas de frecuencias y porcentajes, medias y desviaciones típicas) cuyos resultados presentamos a continuación. Durante el desarrollo de este trabajo se recibieron fondos a través de una ayuda destinada a financiar la contratación predoctoral de personal investigador por la Junta de Castilla y León (EDU/1083/2013), cofinanciada por el Fondo Social Europeo, dentro del Programa Operativo del Fondo Europeo de Desarrollo Regional (FEDER) 2014-2020 de Castilla y León.

Palabras clave: actividades intergeneracionales; acción conjunta intergeneracional; apoyo social percibido; cuestionario; encuesta

\section{ABSTRACT}

Joint intergenerational action (acig). description of intervening variables. In order to know the different types of joint activities carried out by members of different generations, an online 


\section{ACCIÓN CONJUNTA INTERGENERACIONAL (ACIG). DESCRIPCIÓN DE VARIABLES INTERVINIENTES}

questionnaire was administered to a total of 2013 people. Of these, 608 were men and 1405 women. The questionnaire was designed after a review of the instruments used in the field of intergenerational work in various national and international studies. Online, potential participants were contacted to inform them about the purpose of the study and request their participation. The respondents gave their informed consent to participate in the work. Once the data were extracted and codified, the descriptive analyzes were carried out (tables of frequencies and percentages, means and standard deviations), the results of which are presented below. During the development of this work, funds were received through an aid to finance the predoctoral recruitment of research staff by the Junta de Castilla y León (EDU / 1083/2013), co-financed by the European Social Fund, within the Operational Program of the European Regional Development Fund (ERDF) 2014-2020 of Castilla y León.

Keywords: intergenerational activities; joint intergenerational action; perceived social support; questionnaire; poll

\section{INTRODUCCIÓN}

La sociedad actual se encuentra sometida a numerosos factores de cambio,siendo uno de los más llamativos la profunda transformación demográfica percibida cada vez más como un enorme desafío para el siglo XXI (Moreno, Martínez de Miguel y Escarbajal de Haro, 2018). Y es que esta tendencia, afectará a la demanda de los bienes y servicios que necesitará la población en los próximos años (Villalón y Vera, 2012) y, por tanto, las políticas públicas deberán considerar las necesidades adicionales que surjan de las distintas etapas del ciclo vital de los individuos (Rodriguez y Vidal, 2015).De este modo, los conceptos de calidad de vida y envejecimiento activo trascenderán el plano teórico para adentrarse en objetivos de desarrollo práctico, apuntando, en palabras de Del Valle y Coll (2011) "hacia la autonomía y la calidad de vida en su doble vertiente: responsabilidad de acción y compromiso de participación en diferentes espacios sociales".

En nuestro país conocemos la realidad estadística del aumento de la población mayor. Las personas de 65 años y más representan el 18,7\% (8.701.380 personas) de la población, frente al 17,3\% en el año 2011, lo que supuso un incremento de 550.000 individuos. Además de ese colectivo formado por personas mayores de 65 años, el $6 \%$, un tercio aproximadamente sobre el total, tenían 80 años y más, siendo 2.792 .613 habitantes en 2015 (Abellán et al., 2019).Ello, a nuestro parecer, comporta la necesidad explícita de desarrollar espacios propios de participación en los que confluyan diferentes cohortes de la esfera social;participando en programas interactivos e intergeneracionales que suscitan gran interés en la actualidad para la mejora de la calidad de vida, la autoestima y la dignidad de los mayores(Baschiera, Deluigi y Luppi, 2014).

En este sentido, promover el desarrollo de instrumentos de evaluación que nos ayuden a comprender de manera efectiva la naturaleza de las relaciones entre generaciones no solo sería de gran utilidad, sino algo plenamente necesario para hacer uso de estas herramientas en la gran cantidad de contextos educativos que apuestan por la educación intergeneracional en la formación del profesorado y el desarrollo de proyectos basados en la transmisión de conocimientos y experiencias entre jóvenes y mayores (Martínez y Rodríguez, 2017; Sánchez, Sáez, Campillo, y Díaz, 2017). Es por ello, que con el objetivo de conocer los diferentes tipos de actividades conjuntas realizadas por miembros de distintas generaciones diseñamos un instrumento online Acción Conjunta Intergeneracional ( $A C I G)$, el cual, a través de 6 escalas y 14 sub-escalas analiza la información aportada por personas de todas las edades en relación a sus datos demográficos, el apoyo social que las mismas perciben, las actividades conjuntas intergeneracionales presenciales y virtuales que realizan con familiares, amigos, conocidos o profesionales, y una serie de variables psicosociales que nos 
ayudarán a estudiar los beneficios derivados del desarrollo de este tipo de acciones, así como a elaborar unas pautas precisas de intervención en el desarrollo de futuros programas 0 actividades conjuntas entre generaciones.Parte de los datos descriptivos obtenidos por medio de este instrumento se presentan a continuación en el presente estudio.

\section{METODOLOGíA}

El cuestionario, descrito en la Tabla1, fue diseñado para su aplicación a través de la herramienta on-line Survey Monkey, y se adaptó a las previsibles características y necesidades de todos y cada uno de los grupos de población estudiados, siendo sus respuestas totalmente confidenciales y solo utilizables a efectos estadísticos globales, contando el instrumento con la aprobación del Comité de Ética de la Universidad de León. La duración aproximada para la cumplimentación del mismo oscila entre los 25 y 30 minutos.

TABLA I. Descripción del Instrumento ACIG

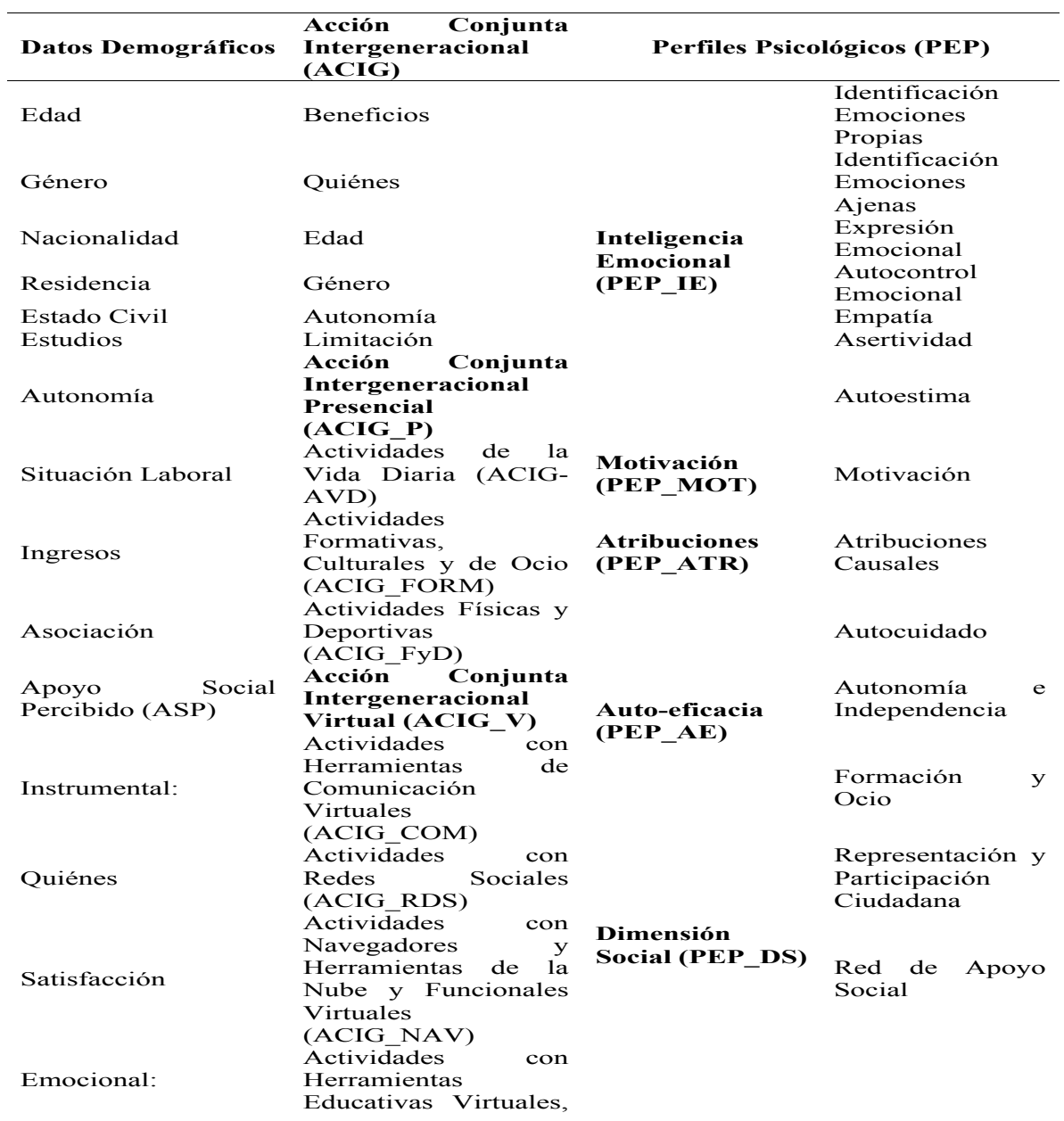




\section{ACCIÓN CONJUNTA INTERGENERACIONAL (ACIG). DESCRIPCIÓN DE VARIABLES INTERVINIENTES}

Una vez validado el instrumento y recogidos los datos por medio de la plataforma de acceso por Internet, los datos cualitativos que se aportan se han descrito por medio de la frecuencia de ocurrencia y el porcentaje.

\section{RESULTADOS}

La muestra original con la que se inició el trabajo $(N=2013)$ queda caracterizada por una edad media de 33.96 años $(D S=16.008)$. La edad es una variable marcadamente no normal $(S-W=.863$, $p \leq .001$ ). La caracterización de la muestra (datos sociodemográficos) queda definida por los siguientes valores (Tabla II).

TABLA II. Datos Demográficos

\begin{tabular}{|c|c|c|}
\hline Variables & Frecuencia & $\%$ \\
\hline \multicolumn{3}{|l|}{ Género } \\
\hline Hombre & 608 & 30.2 \\
\hline Mujer & 1405 & 69.8 \\
\hline \multicolumn{3}{|l|}{ Nacionalidad } \\
\hline Española & 1907 & 94.7 \\
\hline Extranjera & 67 & 3.3 \\
\hline Española y otra & 38 & 1.9 \\
\hline \multicolumn{3}{|l|}{ Residencia } \\
\hline Casa propia & 1541 & 76.6 \\
\hline Casa de familiar & 316 & 15.7 \\
\hline Casa de amigo & 36 & 1.8 \\
\hline Centro o residencia & 120 & 6.0 \\
\hline \multicolumn{3}{|l|}{ Estado Civil } \\
\hline Soltero & 1024 & 50.9 \\
\hline Casado o emparejado & 761 & 37.8 \\
\hline Viudo & 23 & 1.1 \\
\hline Separado & 25 & 1.2 \\
\hline Divorciado & 56 & 2.8 \\
\hline \multicolumn{3}{|l|}{ Nivel de Estudios } \\
\hline E.primarios & 20 & 1.0 \\
\hline E.secundarios & 210 & 10.4 \\
\hline Formación profesional & 138 & 6.9 \\
\hline E.universitarios & 1645 & 81.7 \\
\hline \multicolumn{3}{|l|}{ Autonomía } \\
\hline Solo & 1705 & 84.7 \\
\hline Con ayuda de familiares & 253 & 12.6 \\
\hline Con ayuda profesionales & 12 & .6 \\
\hline Con ayuda de otras personas & 43 & 2.1 \\
\hline
\end{tabular}

\section{Fuente: Elaboración propia}

Con el afán de que estas variables produzcan una mayor eficacia y con la intención de simplificar su interpretación y su manejo, podrán ser objeto de una recodificación en futuros estudios, reagrupando las mismas en un menor número de subcategorías. 
TABLA III. Apoyo Social Percibido (ASP)y realización de diversas Actividades Conjuntas Intergeneracionales

\begin{tabular}{lccc}
\hline Variables & & Frecuencia & \% \\
\hline Recibe apoyo instrumental & No & 1764 & 87.6 \\
& Sí & 86 & 4.3 \\
Recibe apoyo emocional & & 1016 & 50.5 \\
& No & 811 & 40.3
\end{tabular}

Realiza actividades de la vida diaria con personas de otra generación

$\begin{array}{ccc}\text { No } & 1195 & 59.4 \\ \text { Sí } & 431 & 21.4\end{array}$

Realiza actividades culturales, formativas, de ocio, con personas de otras generaciones

$\begin{array}{rrr}\text { No } & 931 & 46.2 \\ \text { Sí } & 689 & 34.2\end{array}$

Realiza actividades deportivas con otra generación

$\begin{array}{rrr}\text { No } & 768 & 38.2 \\ \text { Sí } & 670 & 33.3\end{array}$

Utiliza herramientas de comunicación con otra generación

$\begin{array}{ccc}\text { No } & 408 & 20.3 \\ \text { Sí } & 895 & 44.5 \\ \text { ración } & & \\ \text { No } & 695 & 34.5 \\ \text { Sí } & 417 & 20.7\end{array}$

Emplea navegadores, herramientas de la nube o virtuales con otra generación

$\begin{array}{rrr}\text { No } & 790 & 39.2 \\ \text { Sí } & 239 & 11.9\end{array}$

Emplea herramientas educativas virtuales con otra generación

$\begin{array}{rll}\text { No } & 784 & 38,9 \\ \text { Sí } & 205 & 10,2\end{array}$

Fuente: Elaboración propia

Se observa como el $87.6 \%$ de las personas encuestadas no recibe apoyo instrumental ( $N=1764)$, mientras que el $40.3 \%$ recibe apoyo emocional $(N=811)$. Además, cabe destacar que, para todos los tipos de actividad, el porcentaje de personas que no realizan dicha tarea con miembros de otras generaciones siempre es mayor que el de aquellas que si las realizan, exceptuando el caso del empleo de herramientas de comunicación virtuales (skype, whatsapp, correo electrónico, etc.) dónde el $44.5 \%$ de los participantes $(N=895)$ si las utilizan con personas de diferentes edades frente a un $20.3 \%(N=408)$ que no lo hacen.

\section{DISCUSIÓN Y CONCLUSIONES}

A lo largo de este artículo se han presentado algunos de los resultados descriptivos obtenidos por medio de la implementación de la encuesta online Acción Conjunta Intergeneracional (ACIG),que persigue la obtención de información sobre el tipo de actividades conjuntas que realizan entre si per- 


\section{ACCIÓN CONJUNTA INTERGENERACIONAL (ACIG). DESCRIPCIÓN DE VARIABLES INTERVINIENTES}

sonas de distintas generaciones, así como su relación con diferentes tipos de variables psicosociales, lo que contribuirá en futuros trabajos a contar con información relevante para el diseño de programas y pautas precisas de intervención que mejoren el bienestar y la calidad de vida de los participantes en este tipo de acciones.

De este modo, a uno de los escasos ejemplos de escalas diseñadas para medir los efectos de las interacciones entre jóvenes y mayores participantes en actividades conjuntas intergeneracionales, la Intergenerational Observation Scale (IOS) con la que Jarrott, Smith y Weintraub (2008) lograron establecer una medida confiable de las interacciones sociales durante el desarrollo de diferentes programas intergeneracionales, se le suma el valor de la información que podrá ser obtenida a través de muestra herramienta, en un campo especialmente complicado, al tener que ocuparse de todas las edades posibles y todas las transiciones posibles en prácticamente todos los dominios de la vida (Sánchez y López, 2017).

El paso siguiente, como es lógico, será un análisis relacional entre las variables descritas, de manera que permita dirimir las relaciones que sean más estables.

\section{REFERENCIAS BIBLIOGRÁFICAS}

Abellán García, A., Aceituno Nieto, P., Pérez Díaz, J., Ramiro Fariñas, D., Ayala García, A., y Pujol Rodríguez, R. (2019). Un perfil de las personas mayores en España, 2019. Indicadores estadísticos básicos. Madrid, Informes Envejecimiento en red.

Baschiera, B., Deluigi, R. y Luppi, E. (2014). Educazioneintergenerazionale. Prospettive, progetti e metodologie didáctico-formative per promovere la solidaritáfra le generazioni. Milano: F. Angeli.

Del Valle, G., y Coll, L. (2011). Relaciones Sociales y Envejecimiento Saludable. Institut de l'Envelliment de la UniversitatAutònoma de Barcelona FICE-UAB: FundacióAgrupacióMútua.

Jarrott, S.E., Smith, C.L., y Weintraub, A.P.C. (2008). Development of a St a ndardized To olfor Intergeneration a I Program in g: ThelntergenerationalObservationScale. Journal of IntergenerationalRelationships, 6(4), 433447. doi: $10.1080 / 15350770802474595$

Martínez Heredia, N., y Rodríguez García, A. (2018). Educación intergeneracional: un nuevo reto para la formación del profesorado. Revista de Estudios y Experiencias en Educación, 17(33), 113124.doi: 10.21703/rexe.20181733nmartinez7

Moreno Abellán, P., Martínez de Miguel, L., yEscarbajal de Haro, A. (2018). El impacto educativo de los programas intergeneracionales: un estudio desde la escuela y las diferentes instituciones sociales implicadas. Revista Iberoamericana de Educación, 77(2), 31-54. ISSN: 1022-6508

Rodríguez, M.C., y Vidal Figueroa, C. (2015). Solidaridad intergeneracional: jóvenes y adultos mayores en estrecha colaboración. ProsPectiva. revista de trabajo social e intervención social, 20 , 261-278.

Sánchez, M. y López Doblas, J. (2017). Presente y futuro de la sociología de la vejez en España. Conclusiones de un estudio Delphi. Revista Internacional de Sociología 75(2) doi: http://dx.doi. org/10.3989/ris.2017.75.2.15.44

Sánchez, M., Sáez, J., Campillo, M., y Díaz, P. (2017). Aportación de las personas mayores en los colegios intergeneracionales. La experiencia del proyecto ISCI. Revista de Estudios e Investigación en Psicología y Educación, 5. doi: https://doi.org/10.17979/reipe.2017.0.05.2846

Villalón, G., y Vera, S. (2012). Panorama demográfico en Chile contemporáneo: desafíos para la sociedad del siglo XXI. Revista Anales, 7(3), 35-63. 